\title{
Feasibility and validity of Ecological Momentary Assessment in patients with acute coronary syndrome
}

\author{
François A. M. Jean ${ }^{1}$, Igor Sibon ${ }^{2}$, Mathilde Husky ${ }^{3}$, Thierry Couffinhal| ${ }^{4}$ and Joel Swendsen ${ }^{5,6^{*}}$ (i)
}

\begin{abstract}
Background: In recent years, Ecological Momentary Assessment (EMA) has expanded rapidly in healthcare research but its application specifically to the field of cardiology has been limited. This study presents essential information concerning the feasibility and validity of EMA in patients with acute coronary syndrome.

Methods: Four months after a first-ever acute coronary syndrome, 47 patients completed EMA five times a day for seven consecutive days concerning their current activities, mood and perceived negativity or positivity of daily events.

Results: Compliance with the repeated electronic assessments was high, and no evidence was found for timedependent biases such as fatigue or practice effects. The resulting EMA data were found to have high internal validity, high reliability when considering average scores, and low reliability when considering within-person variance.

Conclusions: We found evidence for the feasibility and intrinsic validity of EMA in patients with acute coronary syndrome. Research examining daily life experiences, symptoms and therapeutic adherence in this population can be reinforced through the use of mobile technologies.
\end{abstract}

Keywords: Acute coronary syndrome, Ecological Momentary Assessment, Validity, Feasibility

\section{Background}

Ambulatory monitoring techniques such as Ecological Momentary Assessment (EMA) have been validated in the investigation of a wide range of diseases and disorders [1-5] as well as in samples ranging from young adolescents to the elderly [6-8]. The contributions of this approach include its capacity to study phenomena in ecologically-valid contexts of daily life, and its ability to collect real-time data overcomes retrospective memory biases associated with traditional and non-electronic research instruments $[9,10]$. Importantly, EMA can also be used to provide real-time therapeutic interventions including medication reminders and specific exercises at

\footnotetext{
*Correspondence: Joel.Swendsen@u-bordeaux.fr

${ }^{5}$ Centre national pour la recherche scientifique (INCIA, CNRS 5287), 146 rue Léo Saignat, Bordeaux, France

Full list of author information is available at the end of the article
}

the moments that they are most needed by patients [11, 12].

It is well-documented that behavioral and psychological variables play a role in the onset as well as recovery from heart disease [13-15], and such variables can have an important impact on the degree to which patients engage in rehabilitation [16]. Ambulatory monitoring of these variables provides an important source of information for both research and clinical intervention. EMA has previously been used in community samples to monitor risk for cardiovascular disease [17], mobile health interventions following cardiac events $[18,19]$, and non-computerized versions of EMA have been used to examine how mood patterns affect coronary artery calcification [20]. However, despite extensive research examining EMA methodology in other health domains, information is currently lacking concerning the basic metrics of 
feasibility and validity in cardiology. The reliance of EMA on repeated daily assessments for periods ranging from days to weeks may raise questions relative to its acceptability among certain patients, as well as concerning the typical compliance rate with the multiple daily assessments. In particular, fatigue effects defined as the decline in performance on a prolonged or demanding task [21] have received little attention to date in EMA applications for cardiology, and it is further unknown if practice effects (improved performance due to repeated assessments) or reactive effects (changes in the frequency or intensity of variables as a function repeated assessment) may constitute important biases in this population. In addition to the question of feasibility and biases, information concerning the reliability and validity of data acquired by EMA is essential for encouraging the use of this approach for research purposes and it is a prerequisite to advancing applications for real-time interventions.

In patients with acute coronary syndrome (ACS), the current study uses EMA over a one-week period to examine daily life experiences and activities. The specific objectives are to: (1) estimate initial study acceptance rates as well as compliance with EMA's multiple daily assessments; (2) examine potential biases and reactive effects associated with the EMA methodology on mood, behavioral and environmental variables; and (3) investigate the split-half reliability adapted to EMA data, as well as internal validity among EMA variables by examining their patterns of association.

\section{Methods}

\section{Participants}

Participants in the present investigation were drawn from a larger study in the cardiology service of a teaching hospital in Bordeaux, Southwest France, that included both an MRI examination and neuropsychological assessments. Participants in the parent study were recruited four months after being admitted for a first-ever acute coronary syndrome. Inclusions were made between September 2009 and December 2012. Persons were excluded if they were younger than 30 years of age, had contraindications for MRI, or if they had a history of neurological disease, major depression, aphasia, or visual, motor or intellectual deficiency preventing the use of the monitoring device. This investigation was approved by the national ethics board and all patients provided written consent to participate. The study protocol conforms to the ethical guidelines of the 1975 Declaration of Helsinki.

\section{Procedure}

Four months after their initial hospitalization, eligible participants were invited to attend a 10 -min training session on computerized EMA including the use of a personal digital assistant (PDA) programmed using a modified version of the Purdue Momentary Assessment Tool version 2.1.2. The research assistant ensured that participants understood each question and response choices displayed on the PDA screen during the ambulatory monitoring week. Following this, participants were given a PDA to carry with them for a one-week period. Each PDA was programmed to administer 5 electronic interviews per day. The signals (an auditory alarm) occurred within each of the following time periods: 9:00 am to $11: 00 \mathrm{am} ; 11: 00$ am to $2: 00 \mathrm{pm} ; 2: 00 \mathrm{pm}$ to $5: 00 \mathrm{pm}$; 5:00 pm to $8: 00 \mathrm{pm}$; and 8:00 pm to $10: 00 \mathrm{pm}$. Assessment times were fixed for each participant, and randomized across participants.

\section{Measures}

At each EMA assessment, participants were asked to describe their current location (being at home, friend's home, partner's home, relative's home, medical institution, work, garden, shop, restaurant, administration, other place inside, other place outside), their social company (being alone, being with a friend, partner, relative, colleague, unknown people, pet, or other company), and their activity (work, sport, television, listening to music, in-person conversation, phone or internet conversation, sports or physical leisure, non-physiscal leisure, hygiene, eating, waiting, resting, cooking, shopping, no activity, or other activity). Response options were provided to participants based on items validated in previous EMA investigations [4] and shown to lack reactive effects when questions are presented in the same order at each assessment. Participant were also asked at each assessment to describe their current degree of happy mood, sad mood, anxious mood, anhedonia and perceived negativity or positivity of daily events using a 7-point scale ranging from -3 ('extremely negative') to 3 ('positive'), in addition to other questions (not analyzed in the current study). Verbatim wording of the full 17-question set and response options are available in supplementary material,Additional file 1: Table S1. The questions were administered in the same order at each assessment and were designed to be completed in 2 min on average. In order to minimize the extent to which responses were influenced by retrospective recall biases, analyses in all samples were limited to assessments that were completed within 20 min of the pre-programmed PDA signal.

\section{Statistical analyses}

We estimated compliance rates as the average number of EMA assessments completed out of all administered assessments. Fatigue effects, practice effects, and reactivity were examined by assessing the frequency, intensity or change in variables (outcomes) a function of study 
duration (predictor variable). In order to study fatigue effects, we created a dichotomous outcome variable to indicate missing data (coded as ' 1 ', compared to completed assessments coded as ' 0 '). Practice effects were examined relative to the time needed to complete each EMA assessment in milliseconds (outcome variable). In order to examine reactivity effects, three representative categories were selected based on their relevance and sufficient frequency of occurrence (present in 5\% or more of all responses) separately for each domain of activity, environmental context and social contact. For this, we decomposed multinomial variables into individual binary outcome variables, coding ' 1 ' for the presence of the category and ' 0 ' for all other responses. To assess reliability, we calculated the averages for happy mood, sad mood, anxiety, anhedonia and perceived negativity/positivity of events for each participant and separately for even and odd days, and then computed a permutation pearson correlation to examine their associations. We also calculated the within-person reliability by focal reliability measures (Rc; [22, 23]), with 95\% confident intervals and bootstrapping using 10,000 iterations. Internal validity was evaluated through the examination of patterns of associations that would be expected among similar or opposing emotions (e.g. sad and anxious mood were expected to be positively correlated; sad and happy were expected to be negatively correlated). EMA data were analyzed using multilevel models with Hierarchical Linear and Nonlinear Modeling Version 6.08 [24]. Meansas-outcomes (gaussian) models were used for continuous outcomes and Bernoulli (binomial logistic) models for dichotomous outcomes. We used two levels for all models (repeated EMA observations at level 1 being nested within individuals at level 2) with robust standard errors and a random effect of participants to correct dependence due to repeated measures. Happy mood, sad mood, anxious mood, anhedonia and perceived negativity/positivity of events were group-mean centered when used as independent variables (i.e. centered around the individual's own mean).

\section{Results}

\section{Participants}

Clinical characteristics of the sample and EMA variables are presented in Table 1. The sample was composed of 47 individuals with a mean age of 54.1 years $(\mathrm{SD}=7.38)$, and $19.1 \%$ were women. Regarding the nature and treatment of ACS, 59.6\% were found to have an ST segment elevation on ECG and $87.2 \%$ were treated with coronary stenting. Participants experienced happy mood at a greater average intensity $(\mathrm{M}=4.4, \mathrm{SD}=1.05)$ than either $\operatorname{sad}(M=1.9, S D=1.43)$ or anxious $\operatorname{mood}(M=1.87$, $\mathrm{SD}=1.39$ ), while anhedonia and perceived negativity/

\begin{tabular}{|c|c|c|c|c|}
\hline & M & $\mathbf{N}$ & SD & $\%$ \\
\hline Age & 54.1 & & 7.38 & \\
\hline Sex: female & & 9 & & 19.1 \\
\hline Troponin (ng/mL) & 34.0 & & 48.1 & \\
\hline ST segment elevation & & 28 & & 59.6 \\
\hline Coronary stenting & & 41 & & 87.2 \\
\hline Coronary bypass & & 2 & & 4.3 \\
\hline \multicolumn{5}{|l|}{ EMA } \\
\hline Happy mood & 4.40 & & 1.05 & \\
\hline Sad mood & 1.90 & & 1.43 & \\
\hline Anxious mood & 1.87 & & 1.39 & \\
\hline Anhedonia & 3.91 & & 1.53 & \\
\hline Perceived stress & 3.73 & & 1.29 & \\
\hline \multicolumn{5}{|l|}{ Location } \\
\hline At home & & 799 & & 71.3 \\
\hline Being outside & & 59 & & 5.3 \\
\hline At relative's home & & 59 & & 5.3 \\
\hline \multicolumn{5}{|l|}{ Social interaction } \\
\hline Alone & & 330 & & 29.5 \\
\hline With family & & 475 & & 42.2 \\
\hline With friends & & 65 & & 5.8 \\
\hline \multicolumn{5}{|l|}{ Activity } \\
\hline Watching television & & 250 & & 22.4 \\
\hline Talking & & 64 & & 5.8 \\
\hline Eating & & 154 & & 13.8 \\
\hline
\end{tabular}

positivity of events were experienced at moderate levels $(\mathrm{M}=3.91, \mathrm{SD}=1.53$, and $\mathrm{M}=3.71, \mathrm{SD}=1.29$, respectively). Concerning behavioral variables, participants were most frequently at home $(71.3 \%)$, with family (42.2\%), and watching television (22.4\%).

\section{Feasibility of EMA}

While approximately half (45.4\%) of eligible participants agreed to take part in the parent study that included MRI, and all of these individuals also agreed to participate in the EMA assessments. No mobile devices were lost or damaged during the study. Overall compliance with the numerous EMA assessments was high, with $79.3 \%(\mathrm{M}=27.76, \mathrm{SD}=4.16)$ of the 35 programmed assessments being completed throughout the week, and and $80.9 \%$ of participants responded to more than $50 \%$ of assessments.

\section{Biases associated with EMA duration}

As demonstrated by Table 2, no fatigue effects were observed in that missing data did not increase as a function of study duration $(\gamma=0.102 ; \mathrm{t}=0.969 ; p>0.05)$. 


\begin{tabular}{|c|c|c|c|}
\hline & $\gamma$ & SE & T ratio \\
\hline Fatigue effects & 0.102 & 0.105 & 0.969 \\
\hline Practice effects & $-13,902.557$ & 7971.901 & -1.744 \\
\hline \multicolumn{4}{|l|}{ Reactive effects } \\
\hline \multicolumn{4}{|l|}{ Location } \\
\hline At home & -0.002 & 0.147 & -0.011 \\
\hline Being outside & 0.214 & 0.147 & 1.455 \\
\hline At relative's home & -0.015 & 0.120 & -0.128 \\
\hline \multicolumn{4}{|l|}{ Social interaction } \\
\hline Alone & 0.117 & 0.103 & 1.134 \\
\hline With family & -0.138 & 0.088 & -1.570 \\
\hline With friends & -0.059 & 0.080 & -0.737 \\
\hline \multicolumn{4}{|l|}{ Activity } \\
\hline Watching television & -0.080 & 0.117 & -0.680 \\
\hline Talking & -0.173 & 0.158 & -1.086 \\
\hline Eating & 0.160 & 0.092 & 1.740 \\
\hline \multicolumn{4}{|l|}{ Mood states } \\
\hline Happy mood & 0.116 & 0.074 & 1.576 \\
\hline Sad mood & -0.118 & 0.111 & -1.061 \\
\hline Anxious mood & -0.139 & 0.114 & -1.223 \\
\hline Anhedonia & 0.013 & 0.004 & 0.140 \\
\hline Perceived stress & -0.209 & 0.097 & $-2.161^{*}$ \\
\hline
\end{tabular}

${ }^{*} p<0.05$. Fatigue $=$ number of missing observation by day of study; Practice $=$ milliseconds needed to complete EMA by day of study; Reactivity $=$ frequency or intensity of variables by day of study

Practice effects were also absent as participants did not respond more rapidly to daily life assessments as the study progressed $(\gamma=-13,902.557 ; \mathrm{t}=-1.744$; $p>0.05$ ). Concerning reactivity (alterations in variable intensity or frequency as a function of repeated assessments), no effects were observed for happy mood $(\gamma=0.116 ; \mathrm{t}=1.576 ; p>0.05) ; \operatorname{sad} \operatorname{mood}(\gamma=-0.118$; $\mathrm{t}=-1.061 ; \quad p>0.05) ; \quad$ anxious $\operatorname{mood}(\gamma=-0.139$; $\mathrm{t}=-1.223 ; p>0.05)$ or anhedonia $(\gamma=0.013 ; \mathrm{t}=0.004$; $p>0.05)$. However, the perceived negativity/positivity of events changed as the study progressed $(\gamma=-0.209 ; \mathrm{t}=-2.161 ; p<0.05)$. Concerning reactivity, no significant change was observed as a function of repeated assessments with regard to activity (talking: $\gamma=-0.173 ; \mathrm{t}=-1.086 ; p>0.05$; eating: $\gamma=0.160$; $\mathrm{t}=1.740 ; \quad p>0.05 ;$ watching television: $\gamma=-0.080$; $\mathrm{t}=-0.680$ ), social company (being alone: $\gamma=-0.117$; $\mathrm{t}=1.134 ; p>0.05$; being with friends: $\gamma=-0.059$; $\mathrm{t}=-0.737 ; p>0.05$; being with family: $\gamma=-0.138$; $\mathrm{t}=-1.570 ; p>0.05)$, or for environmental contexts (being at home: $\gamma=-0.002 ; \mathrm{t}=-0.011 ; p>0.05$; being outside: $\gamma=0.214 ; \mathrm{t}=1.455 ; p>0.05$; being at relative's home: $\gamma=-0.015 ; \mathrm{t}=-0.128 ; p>0.05)$.
Table 3 Relationships among EMA variables (internal validity)

\begin{tabular}{lrcc}
\hline & V & SE & T ratio \\
\hline Mood states & & & \\
Sad_happy & -0.333 & 0.071 & $-4.723^{* * *}$ \\
Anhedonia_happy & -0.605 & 0.058 & $-10.378^{* * *}$ \\
Anhedonia_sad & 0.143 & 0.059 & $2.435^{*}$ \\
Anxiety and mood & & & \\
Anxiety—happy & -0.253 & 0.057 & $-4.420^{* * *}$ \\
Anxiety—sad & 0.377 & 0.048 & $7.869^{* * *}$ \\
Anxiety-anhedonia & 0.095 & 0.037 & $2.578^{*}$ \\
Perceived stress and emotions & & & \\
Perceived stress-happy & -0.396 & 0.067 & $-5.924^{* * *}$ \\
Perceived stress-sad & 0.0260 & 0.053 & 0.495 \\
Perceived stress-anhedonia & 0.222 & 0.040 & $5.579^{* * *}$ \\
Perceived stress-anxiety & 0.104 & 0.053 & 1.957 \\
\hline
\end{tabular}

${ }^{*} p<0.05 ;{ }^{* * *} p<0.001$

\section{Reliability and internal validity}

In order to assess reliability, all continuous EMA variables were averaged for each participant across even and odd days. These scores for each participant were strongly correlated for happy mood $(\mathrm{r}=0.79, \mathrm{t}=8.7, p<0.001)$, sad mood $(\mathrm{r}=0.95, \mathrm{t}=19.8, p<0.001)$, anxiety $(\mathrm{r}=0.83$, $\mathrm{t}=9.9, p<0.001)$, anhedonia $(\mathrm{r}=0.78, \mathrm{t}=8.3, p<0.001)$ and perceived negativity/positivity of events $(\mathrm{r}=0.87$, $\mathrm{t}=11.9, p<0.001)$. The patterns of association among ambulatory monitoring variables are presented in Table 3 . As expected, happy mood was negatively associated with $\operatorname{sad} \operatorname{mood}(\gamma=-0.333 ; \mathrm{t}=-4.723 ; p<0.001)$ and, conversely, increases in anxiety were associated with greater $\operatorname{sad} \operatorname{mood}(\gamma=0.377 ; t=7.869 ; p<0.001)$. No association was observed between perceived negativity/positivity of events with either anxious $(\gamma=0.104 ; \mathrm{t}=1.957 ; p>0.05)$ or sad mood $(\gamma=0.0260 ; \mathrm{t}=0.495 ; p>0.05)$. The focal reliability coefficients were low for all emotions: happy $\mathrm{Rc}=0.18(0.110 .25)$, sad $\mathrm{Rc}=0.33(0.26-0.40)$, anhedonia $\mathrm{Rc}=0.07(0.01-0.14)$, anxiety $\mathrm{Rc}=0.29(0.22-0.36)$, perceived negativity/positivity $\mathrm{Rc}=0.18(0.11-0.25)$.

\section{Discussion}

Despite increasing interest in Ecological Momentary Assessment in the field of cardiovascular disease, information concerning its basic feasibility and validity has been lacking. The present findings provide strong support for the feasibility and validity of this methodology in ACS patients.

Before the concepts of reliability or validity can be examined, it is first necessary that EMA be acceptable to patients, measurable both in terms of initial study 
acceptance as well as compliance with the repeated daily assessments. In the current sample, all eligible participants agreed to participate. It is notable that this study was part of a broader investigation involving MRI, and therefore it was not possible to examine the initial participation acceptance rate independently from the other methodologies involved. However, a high overall compliance rate with the repeated daily assessments was also high (79.3\%). This rate is similar to what has been observed in healthy samples and in diverse psychiatric populations [4]. Together, these elements provide fundamental support for establishing the feasibility of this methodology for individuals discharged after cardiac events.

The present study showed no fatigue effects, a finding similar to what has been demonstrated in a variety of clinical populations $[3,4]$. In addition, no practice effects were found, suggesting that the time it takes participants to complete each assessment did not vary as a function of study duration. Finally, while mood levels were not altered throughout the assessment period, perceived event negativity/positivity changed significantly with study duration. This finding differs from what has been observed with psychiatric samples [3], but may be explained by the fact that the initiation of the study occurred at the time of a scheduled clinical evaluation and it is possible that event perceptions change when patients returned home and to their regular daily routines. However, should reactivity to the repeated daily assessments be observed in future investigations, researchers may wish to control for day of study when conducting analyses of EMA data.

In light of findings supporting the feasibility of EMA and the general absence of time-dependent biases, a final issue concerns the reliability and validity of the resulting data. There are numerous forms of reliability and validity and therefore final recommendations should be based on multiple studies and methods. This particular study examined reliability first by calculating correlations for a given variable assessed across alternating days, and assessed internal validity by examining the presence of correlations in expected directions among parallel or opposed mood states. Strong reliability was observed for all variables examined, and the results also confirm the internal validity of EMA data through the expected correlations among daily life variables, as has been reported in other samples using this technique [4]. However, using the Rc approach that examined within-person variability, low reliability was found for all emotions and the perceived negativity/positivity of events. These findings underscore the fact that these variables are highly fluctuating phenomena, and although their average levels may be reliable, methods such as EMA are necessary for detecting such momentary fluctuations.
These findings should be interpreted in light of several characteristics of the sample and its methodology. First, the moderate sample size may not have been sufficient to detect small but clinically significant effects. While the higher prevalence of acute coronary syndrome in men is also reflected in the current sample, replicating these findings in samples including a larger percentage of women would permit the examination of potential sex differences. In comparison with other studies [25], there was a high proportion of ST elevation in our sample. This may be explained by the fact that very few hospitals with equipment to perform coronary angiography exist in the area where the study was conducted, and therefore patients with ST elevation were referred to this teaching hospital at greater proportions than in other areas. It is also notable that perceived event negativity or positivity was not significantly associated with negative mood states, a finding that may be due to the bimodal scale used for this variable that was different from the unidirectional scales used for mood states. Finally, it should be noted that this investigation did not examine concurrent validity between EMA and traditional self-report assessments commonly used in cardiology. Again for this reason, final conclusions regarding reliability and validity should be based on a diversity of studies using different methods.

\section{Conclusions}

ACS patients demonstrated highly-satisfactory compliance with the repeated assessments provided by EMA, supporting the global feasibility of this methodology in this population. The data generated from this technique were also psychometrically sound, with evidence for reliability as well as internal validity and a general absence of timedependent biases. A reality in the field of cardiology is that the vast majority of progress made by patients depends on their behavior when they are not accompanied by a clinician. It is indeed during their daily lives that they must remember to take their medications, to avoid risk factors and to practice prescribed exercises. In this way, the present findings strongly suggest that EMA can be used not only for research purposes but also in conjunction with standard treatment to improve medication adherence or to deliver psychosocial interventions. These latter applications are a growing subject of interest, and the lifestyle changes needed after an acute coronary syndrome may therefore be of particular importance for future EMA studies.

\section{Supplementary information}

Supplementary information accompanies this paper at https://doi. org/10.1186/s12872-020-01774-w.

Additional file 1. Original French-language EMA questions and response items 


\section{Abbreviations}

ACS: Acute Coronary Syndrome; EMA: Ecological Momentary Assessment; PDA: Personal Digital Assistant.

\section{Acknowledgements}

The authors are grateful for the contributions of S. Lassalle-Lagadec, S. Ledure, P. Perez, J. Asselineau and R. Sitta.

\section{Authors' contributions}

FJ collected and analyzed the EMA data. IS, TC, MH and FJ contributed to writing the manuscript. JS designed the study, and contributed to data analysis and writing the manuscript. All authors read and approved the final manuscript.

\section{Funding}

This work was supported by the French National Center for Scientific Research (Pr. Swendsen; UMR5287) and the Bordeaux University Hospital (Pr. Sibon; IF2007-31). The funding bodies played no role in the design of the study, the collection, analysis, or interpretation of the data, or in the writing the manuscript and the decision to submit for publication.

\section{Availability of data and material}

The datasets generated and/or analyzed during the current study are not publicly available due French regulations for health care research, but are available from the corresponding author upon reasonable individual requests.

\section{Ethics approval and consent to participate}

This investigation was approved by the Ethics Committee for the Protection of Individuals in Research (CPP) of Southwest France and French Overseas Territories, and all patients provided written consent to participate. The study protocol conforms to the ethical guidelines of the 1975 Declaration of Helsinki.

\section{Consent for publication}

Not applicable.

\section{Competing interests}

The authors declare that they have no competing interests.

\section{Author details}

${ }^{1}$ Department of Psychiatry, Dr Jean Eric Techer Hospital, Calais, France. ${ }^{2}$ Centre hospitalier universitaire de Bordeaux, Bordeaux, France. ${ }^{3}$ Université de Bordeaux, Bordeaux, France. ${ }^{4}$ Institut national de la sante et de la recherche medicale (U1034), Bordeaux, France. ${ }^{5}$ Centre national pour la recherche scientifique (INCIA, CNRS 5287), 146 rue Léo Saignat, Bordeaux, France. ${ }^{6}$ Ecole Pratiques de Hautes Etudes, PSL University, Paris, France.

Received: 4 February 2020 Accepted: 8 November 2020

Published online: 27 November 2020

\section{References}

1. Stone AA, Shiffman S. Ecological momentary assessment (EMA) in behavorial medicine. Ann Behav Med. 1994;16(3):199-202.

2. Granholm E, Loh C, Swendsen J. Feasibility and validity of computerized ecological momentary assessment in schizophrenia. Schizophr Bull. 2008;34(3):507-14.

3. Husky MM, Gindre C, Mazure CM, Brebant C, Nolen-Hoeksema S, Sanacora $\mathrm{G}$, et al. Computerized ambulatory monitoring in mood disorders: feasibility, compliance, and reactivity. Psychiatry Res. 2010;178(2):440-2.

4. Johnson El, Grondin O, Barrault M, Faytout M, Helbig S, Husky M, et al. Computerized ambulatory monitoring in psychiatry: a multi-site collaborative study of acceptability, compliance, and reactivity. Int J Methods Psychiatr Res. 2009;18(1):48-57.

5. Serre F, Fatseas M, Debrabant R, Alexandre J-M, Auriacombe M, Swendsen J. Ecological momentary assessment in alcohol, tobacco, cannabis and opiate dependence: a comparison of feasibility and validity. Drug Alcohol Depend. 2012;126(1-2):118-23.

6. Cain AE, Depp CA, Jeste DV. Ecological momentary assessment in aging research: a critical review. J Psychiatr Res. 2009;43(11):987-96.
7. Mulvaney SA, Rothman RL, Dietrich MS, Wallston KA, Grove E, Elasy TA, et al. Using mobile phones to measure adolescent diabetes adherence. Health Psychol. 2012;31(1):43-50.

8. Scharf DM, Martino SC, Setodji CM, Staplefoote BL, Shadel WG. Middle and high school students' exposure to alcohol- and smoking-related media: a pilot study using ecological momentary assessment. Psychol Addict Behav. 2013;27(4):1201-6.

9. Broderick JE, Schwartz JE, Shiffman S, Hufford MR, Stone AA. Signaling does not adequately improve diary compliance. Ann Behav Med. 2003;26(2):139-48.

10. Stone AA, Shiffman S, Schwartz JE, Broderick JE, Hufford MR. Patient compliance with paper and electronic diaries. Control Clin Trials. 2003;24(2):182-99.

11. Mundi MS, Lorentz PA, Grothe K, Kellogg TA, Collazo-Clavell ML. Feasibility of smartphone-based education modules and ecological momentary assessment/intervention in pre-bariatric surgery patients. ObesSurg. 2015;25:1875-81.

12. Ruscio AC, Muench C, Brede E, Waters AJ. Effect of brief mindfulness practice on self-reported affect, craving, and smoking: a pilot randomized controlled trial using ecological momentary assessment. Nicotine Tob Res. 2016;18(1):64-73.

13. Dias CMCC, Macedo LB, Gomes LTJC, de Oliveira PLSP, Albuquerque IVS, Lemos AQ, et al. Quality of life of patients after an acute coronary event: hospital discharge. J Clin Med Res. 2014;6(5):362-8.

14. Kronish IM, Rieckmann N, Burg MM, Alcántara C, Davidson KW. The psychosocial context impacts medication adherence after acute coronary syndrome. Ann Behav Med. 2014;47(2):158-64

15. Thombs BD, Bass EB, Ford DE, Stewart KJ, Tsilidis KK, Patel U, et al. Prevalence of depression in survivors of acute myocardial infarction. J Gen Intern Med. 2006;21(1):30-8.

16. Jones MC, Smith K, Herber O, White M, Steele F, Johnston DW. Intention, beliefs and mood assessed using electronic diaries predicts attendance at cardiac rehabilitation: an observational study. Int J Nurs Stud. 2018;88:143-52.

17. Kamarck TW, Schwartz JE, Shiffman S, Muldoon MF, Sutton-Tyrrell K, Janicki DL. Psychosocial stress and cardiovascular risk: what is the role of daily experience? J Pers. 2005;73(6):1749-74.

18. Webber CJ, O'Hea EC, Abar B, Bock B, Boudreaux ED. Ecological momentary assessment and first smoking cessation lapse after an acute cardiac event: a pilot study. J Health Psychol. 2017;25:1076-81.

19. Sengupta A, Beckie T, Dutta K, Dey A, Chellappan S. Usability study of a mobile health intervention system for women with coronary heart disease. JMIR Form Res. 2020;4:e16420.

20. Kroenke $\mathrm{CH}$, Seeman T, Matthews K, Adler N, Epel E. Mood patterns based on momentary assessment of positive and negative moods over a day and coronary artery calcification in the CARDIA study. Psychosom Med. 2012;74(5):526-34.

21. APA Dictionary of Psychology [Internet]. [cité 9 mai 2020]. Disponible sur: https://dictionary.apa.org/fatigue-effect.

22. Bolger N, Laurenceau J-P. Intensive longitudinal methods: an introduction to diary and experience sampling research. New York: Guilford Press; 2013.

23. Shrout P, Lane SP. Psychometrics. In: Mehl MR, Conner TS, editors. Handbook of research methods for studying daily life. New York: Guilford Press; 2012. p. 302-20.

24. Raudenbush SW, Bryk AS, Congdon R. HLM 6 for Windows [Computer software]. Skokie: Scientific Software International, Inc.; 2004.

25. Güntekin Ü, Tosun V, Kilinç AY, Saydam G, Korucuk N, Bozdemir MN. ST segment elevation myocardial infarction (STEMI) patients are more likely to achieve lipid-lowering treatment goals: a retrospective analysis of patients presenting with first acute coronary syndromes. Medicine (Baltimore). 2018;97(39):e12225.

\section{Publisher's Note}

Springer Nature remains neutral with regard to jurisdictional claims in published maps and institutional affiliations. 